\title{
A machine learning approach identifies unique predictors of borderline personality disorder
}

\author{
By Dr. Jessica Edwards
}

Researchers in the USA have identified critical predictors of borderline personality disorder (BPD) in late adolescence, using a machine learning approach. Joseph Beeney and colleagues harnessed data from a large, prospective, longitudinal dataset of $>2,400$ girls who were evaluated yearly for various clinical, psychosocial and demographic factors. Their optimal predictive model included 19 predictors of a possible 128. The top predictors included mood and anxiety symptoms, poor self-control, harsh punishment and poor functioning. These predictors were relatively stable across three key developmental periods - late childhood, early-adolescence and mid-adolescence - suggesting that BPD risk factors do not markedly change over time.

"A key take away from these findings is that there are quite a few early signs of BPD that might signal a need for children and adolescents to engage in preventative treatment", explains Beeney. "While we don't have enough data on what might alter the course of BPD in those with these early risk factors, knowing which risk factors are most influential can help us design better treatments and allow us to intervene early".

Another important finding from this study was that the predictive model for BPD did not substantially overlap with models for depression or conduct disorder (CD). For example, anxiety was a major predictor of BPD symptoms while sensation seeking was a major predictor of CD symptoms. The researchers also found key differences in parenting risk factors between these disorders. The hope is that these data and future research might help direct clinicians towards important risk factors unique to each disorder that might be effectively targeted during treatment.

"Being able to differentiate between early risk for symptoms of BPD, CD and depression in adolescence is important", says Beeney. "Although recent research has suggested that a set of common factors might increase the risk for all psychopathology, our findings suggest that the most important factors for each disorder could be distinct".

\begin{abstract}
Referring to:
Beeney, J.E., Forbes, E.E., Hipwell, A.E., Nance, M., Mattia, A., Lawless, J.M., Banihashemi, L. \& Stepp, S.D. (2020), Determining the key childhood and adolescent risk factors for future BPD symptoms using regularized regression: comparison to depression and conduct disorder. J. Child Psychol. Psychiatr. doi: 10.1111/ jcpp.13269.
\end{abstract}

\section{Glossary:}

\section{Borderline personality disorder:} according to the DSM-5, BPD is diagnosed based on: (1) a pervasive pattern of instability of interpersonal relationships, selfimage, and affects; and (2) marked impulsivity beginning by early adulthood and present in various contexts.

\section{Conduct disorder (CD): CD is} characterized by behaviour that violates either the rights of others or major societal norms, such as aggression, destruction of property or theft. To be diagnosed with conduct disorder, symptoms must cause significant impairment in social, academic or occupational functioning. The disorder is typically diagnosed prior to adulthood. 